\title{
Pesquisa de lasalocida y monensina en leche cruda de vacas lecheras suplementadas con estos ionóforos ${ }^{\#}$
}

\section{Detection of lasalocid and monensin in raw milk samples from supplemented dairy cows}

\author{
A Jerez ${ }^{a^{*}}$, R Chihuailaf $^{\mathrm{b}}$, M Gai $^{\mathrm{c}}$, M Noro $^{\mathrm{d}}$, F Wittwer $^{\mathrm{d}}$ \\ anstituto de Farmacia, Facultad de Ciencias, Universidad Austral de Chile, Valdivia, Chile. \\ ${ }^{\text {b}}$ Escuela de Medicina Veterinaria, Facultad de Recursos Naturales, Universidad Católica de Temuco, Temuco, Chile. \\ ${ }^{\mathrm{c}}$ Facultad de Ciencias Químicas y Farmacéuticas, Departamento de Ciencias y Tecnología Farmacéutica, \\ Universidad de Chile, Santiago, Chile. \\ ${ }^{\mathrm{d}}$ Instituto de Ciencias Clínicas Veterinarias, Facultad de Ciencias Veterinarias, Universidad Austral de Chile, Valdivia, Chile.
}

\begin{abstract}
SUMMARY
The ionophores lasalocid and monensin have been used as food additives to improve body weight gain in beef cattle and milk production in dairy cows. The aims of this work were to detect the presence of these compounds in raw milk samples and their ruminal disappearance rate in dairy cows using HPLC techniques. Two Friesian herds were used. Cows from one herd were supplemented with lasalocid and the other with monensin during 18 and 21 days respectively, and according to the recommended dose $(300 \mathrm{mg} / \mathrm{d})$. Daily raw milk samples were obtained during the supplementing period and the presence of lasalocid and monensin was analysed using HPLC techniques (LOD 30 and 5 ppb respectively). In a parallel trial fluid ruminal samples obtained from fistulised cows up to 24 hours after a single intra ruminal administration with the ionophores were analysed using the same HPLC techniques. Both ionophores were not detected in any of the milk samples during all the experimental period. The low transference of these ionophores to the milk could be related to their expected low absorption rate related to their physical-chemical proprieties. Lasalocid was detected in the ruminal fluid up to 6 hours after administration and monensin up to 4 hours of administration. According to the results, lasalocid and monensin are not detected in raw milk samples from supplemented cows using techniques with detection limits of 30 and 5 ppb, respectively.
\end{abstract}

Key words: ionophores, milk, ruminal fluid, HPLC.

\section{RESUMEN}

Los ionóforos lasalocida y monensina han sido usados como aditivos en el alimento para mejorar la ganancia de peso en ganado de carne y la producción de leche en vacas. Los objetivos de este trabajo fueron detectar la presencia de estos compuestos en muestras de leche cruda y la tasa de desaparición ruminal, utilizando HPLC como técnica analítica. Dos rebaños de vacas Friesian fueron utilizados. Las vacas de cada rebaño fueron suplementadas con lasalocida y monensina por 18 y 21 días, respectivamente, a la dosis recomendada (300 mg/d). Las muestras fueron obtenidas diariamente durante el periodo de suplementación y la presencia de lasalocida y monensina fue analizada usando una técnica HPLC (LOD 30 y 5 $\mathrm{ppb}$, respectivamente). En ensayos paralelos, empleando vacas fistuladas, se obtuvieron muestras de líquido ruminal durante 24 horas posterior a una administración intrarruminal de ambos ionóforos, las que fueron analizadas mediante la misma técnica. Ambos ionóforos no fueron detectados en las muestras de leche obtenidas durante el periodo experimental. La escasa transferencia de estos ionóforos a la leche podría estar relacionada a su baja tasa de absorción esperada acorde con sus propiedades fisicoquímicas. La lasalocida fue detectada en el líquido ruminal por 6 horas postadministración, mientras que para la monensina el tiempo fue de 4 horas. De acuerdo con los resultados no se detecta la presencia de lasalocida o monensina en muestras de leche cruda de vacas suplementadas, usando una técnica con límites de detección de 30 y 5 ppb, respectivamente.

Palabras clave: ionóforos, leche, líquido ruminal, HPLC.

\section{INTRODUCCIÓN}

Los antimicrobianos usados como aditivos en el alimento de los animales de producción han sido por muchos años la categoría de mayor controversia. Los aspectos involucrados al respecto son la generación de resistencia a antibióticos en bacterias de animales y la influencia que puede generar sobre la salud pública de

Aceptado: 28.11.2013.

\# Financiado por Consorcio Lechero, Proyecto M2P8

* alejandrojerez@uach.cl las personas (Gustafson 1991). La industria de la leche ha utilizado por años ionóforos como aditivos en dosis que promueven una mejor eficiencia en la producción (Duffield y Bagg 2000).

En 1968 el término ionóforo fue usado por primera vez en referencia a todos los compuestos poliéteres carboxílicos que se enmarcaban dentro de la definición clásica de antibióticos (Pressman 1968). Estos compuestos de origen natural provienen de productos de fermentación de varios actinomicetos (Duffield y Bagg 2000). Desde la década de 1970 se han utilizado para manipular la fermentación ruminal, mejorando la eficiencia en la utilización del 
alimento y ganancia de peso de rumiantes (Pinos Rodríguez y González Muñoz 2000).

Los ionóforos son compuestos lipofílicos, tóxicos para varias bacterias, protozoos, hongos y organismos superiores (Russell y Mantobani 2002). Su toxicidad depende de la capacidad de penetrar membranas biológicas alterando el flujo de iones desde y hacia la célula. Los ionóforos, como monensina y lasalocida, han sido propuestos para su empleo como aditivos en el alimento de vacas lecheras (Russell y Strobel 1989). Los efectos de los ionóforos sobre las bacterias son alteraciones en el flujo de iones $\mathrm{y}$, consecuentemente, un incremento significativo en la actividad del sistema $\mathrm{Na}^{+} / \mathrm{K}^{+}$y $\mathrm{H}^{+}$ATPasa de la bacteria ruminal para mantener el balance iónico y el pH intracelular, comprometiendo su capacidad de crecimiento y reproducción (Bergen y Bates 1984). La magnitud del daño a la bacteria depende de la sensibilidad de esta al ionóforo, ello se relaciona con la permeabilidad de las membranas a las macromoléculas (Russell 1987). Las bacterias Gram positivas son más sensibles a los ionóforos que las Gram negativas, ya que poseen una estructura menos compleja que estas últimas (Russell y col 1988).

Los ionóforos favorecen las bacterias que producen propionato, optimizando los metabolismos energético y nitrogenado, reduciendo el riesgo de timpanismo y acidosis láctica. Varios de los beneficios demostrados por los ionóforos están asociados a una mejora del estatus energético de la vaca durante la lactancia temprana (Mohamadi y col 2009). Los beneficios incluyen una menor movilización de grasa corporal, evidenciada por menores concentraciones plasmáticas de ácidos grasos no esterificados y cetonas y un incremento de la glucemia. En las vacas hay una menor incidencia de cetosis, desplazamiento del abomaso, menor pérdida en la condición corporal e incremento en la eficiencia en volumen y composición de la producción de leche (McGuffey y col 2001, Gandra y col 2012). Al respecto, se describe que la suplementación con monensina en vacas lecheras disminuye en $0,3 \mathrm{~kg}$ /día el consumo de materia seca y aumenta en 0,7 a $1,5 \mathrm{~kg}$ /día la producción de leche, dependiendo del tipo de dieta (Espíndola 2010).

El empleo de la monensina en el ganado lechero ha sido aprobado desde fines de la década de 1970 en varios países, tales como Australia, Argentina, Nueva Zelanda y Sudáfrica (Duffield y Bagg 2000). En 1999 la UE prohibió el uso de ionóforos en vacas lecheras sobre la base de un principio precautorio. Sin embargo, desde el 2004 la FDA autorizó su uso con fines de producción lechera en los Estados Unidos de América. En Chile se autoriza el uso de monensina por parte del Servicio Agrícola y Ganadero (SAG) con fines de producción de leche, pero no lo hace para la lasalocida.

Hoy existe carencia de métodos accesibles para la determinación de ionóforos en leche, por lo que es necesario disponer de técnicas que permitan controlar el eventual empleo no autorizado de estos productos por parte de ganaderos, para ello este estudio tuvo como objetivo pesquisar ionóforos en la leche de vacas lecheras suplementadas con lasalocida y monensina mediante técnicas cromatográficas.

\section{MATERIAL Y MÉTODOS}

\section{ANIMALES PARA ESTUDIO EN LECHE}

El trabajo fue realizado utilizando vacas pertenecientes a los rebaños del Hospital Veterinario para la lasalocida y en la Estación Experimental Vista Alegre para la monensina, ambos de propiedad de la Universidad Austral de Chile y ubicados en la provincia de Valdivia en la Región de Los Ríos, Chile. El clima de la provincia de Valdivia es templado húmedo con influencia mediterránea, con una temperatura media de $12{ }^{\circ} \mathrm{C}$ y una precipitación anual de $1.871 \mathrm{~mm}$. Fueron utilizados dos grupos de vacas, uno formado por 11 vacas Holstein Friesian para lasalocida y otro compuesto de 10 vacas Holstein Friesian para monensina. Para el caso de la lasalocida se usaron dos vacas como control y para la monensina, una. Para ambos casos las vacas se encontraban entre el $2^{\circ}$ y $6^{\circ}$ mes de lactancia y fueron declaradas clínicamente sanas por un profesional médico veterinario.

\section{ALIMENTACIÓN}

Las vacas fueron mantenidas en pastoreo rotativo de praderas permanentes con predominio de Lolium perenne y Bromus valdivianus, y libre acceso a agua en potreros de 1 a 4 ha de superficie y con sistema de rotación en franjas asignadas dos veces al día. Además, cada animal recibió durante la ordeña de la mañana $(8: 00 \mathrm{~h})$ y tarde $(17: 00 \mathrm{~h})$ $2,5 \mathrm{~kg}$ de concentrado comercial para vaca en lactancia, sin aporte adicional de mezclas minerales.

\section{DOSIS}

La elección de la dosis administrada se realizó a base de un ajuste preexperimental de ella en un periodo de evaluación, donde los animales fueron monitoreados en cuanto al consumo de alimento y eventuales efectos adversos que pudieran padecer, especialmente su impacto en la inhibición de apetito. Para esto se tomó como referencia la dosis recomendada por las empresas fabricantes de estos productos, en donde establecen una dosis máxima recomendada de $300 \mathrm{mg} / \mathrm{vaca} /$ día. Los productos fueron suplementados por 18 días para lasalocida (Bovatec $® 15 \%$, Alpharma) y por 20 días para monensina (Rumensin $® 100$, Elanco).

\section{ADMINISTRACIÓN}

El producto fue mezclado con maíz rolado, el que fue administrado durante la ordeña de la mañana. Para ello, 2,0 gramos de Bovatec $15 \%$ en el ensayo con lasalocida o 3,0 gramos de Rumensin 10\% para el ensayo con monensina fueron mezclados con 200 g de maíz rolado, el que 
se suministró de forma individual a cada vaca previo al suministro del restante concentrado $(2,3 \mathrm{~kg})$, asegurándose de esta forma que las vacas consumieran la totalidad del ionóforo. Las vacas controles recibieron $2,5 \mathrm{~kg}$ de maíz rolado.

\section{MUESTRAS}

Se obtuvieron muestras de leche compuestas de los cuatro cuartos durante la ordeña de la mañana desde el día 0 del estudio y hasta la finalización de este, día 19 para la lasalocida y 21 para monensina. Las muestras de $10 \mathrm{~mL}$ de leche se obtuvieron desde el medidor proporcional (Waikato) ubicado en la línea de leche.

\section{ESTUDIO EN LÍQUIDO RUMINAL}

A dos grupos de tres vacas Holstein Frisian, en lactancia y con fistula ruminal, se les administró de forma intrarruminal lasalocida y monensina, respectivamente. Dos de las vacas fueron dosificadas con $300 \mathrm{mg}$ y la restante con $600 \mathrm{mg}$ para cada grupo. Una cuarta vaca de cada grupo de similares condiciones se mantuvo como control. Muestras de líquido ruminal se obtuvieron a las $0,4,6,8,11,13$ y 24 horas postadministración, en el caso de la lasalocida y la monensina, las que fueron llevadas al laboratorio del Instituto de Farmacia de la Universidad Austral de Chile para su análisis mediante HPLC. Las muestras de líquido ruminal fueron obtenidas del saco caudoventral del rumen mediante compresión y filtración de material ruminal, obteniéndose $15 \mathrm{~mL}$ de muestra en cada oportunidad.

\section{METODOLOGÍA ANALÍTICA}

Se aplicaron métodos HPLC-UV, previamente validados según las especificaciones de la FDA para métodos bioanalíticos (FDA 2001), para la determinación de los ionóforos en muestras de leche cruda bovina (Jerez y col 2013). Las condiciones de análisis se detallan en el cuadro 1.

La extracción, en el caso de ambos compuestos, fue del tipo líquido-líquido y en el procedimiento se tomó una alícuota de $1 \mathrm{~mL}$ de leche cruda, se llevó a un tubo
Falcon® de $20 \mathrm{~mL}$ y se le adicionaron $6 \mathrm{~mL}$ de metanol para desproteinizar. Posteriormente se agitó vigorosamente por 1 minuto de manera manual y luego se llevó a centrífuga a $3.000 \mathrm{rpm}$ por 10 minutos.

Luego se retiró el sobrenadante, se vació a un embudo de decantación de $50 \mathrm{~mL}$, donde se le agregaron $6 \mathrm{~mL}$ de $\mathrm{NaCl} 10 \%$. Posteriormente se extrajo con 2 porciones de $6 \mathrm{~mL}$ de diclorometano (DCM) y se agitó suavemente y se dejó reposar por 15 minutos para la separación de fases.

Se rescató la fase inferior en un tubo de ensayo, y se llevó a sequedad, en baño termorregulado a $45^{\circ} \mathrm{C}$ y con presencia de burbujeo de nitrógeno gaseoso. Una vez seco, se reconstituyó con $1 \mathrm{~mL}$ de una solución 90:10 de metanol:agua para su posterior inyección.

Los límites de detección (LD) de estas metodologías fueron de $30 \mathrm{ng} / \mathrm{mL}$ para lasalocida y de $5 \mathrm{ng} / \mathrm{mL}$ para monensina.

\section{RESULTADOS Y DISCUSIÓN}

En el caso de las muestras de líquido ruminal, solo fue posible detectar la lasalocida hasta las 4 horas en una vaca tratada con $300 \mathrm{mg}$ y hasta las 6 horas en el animal que recibió $600 \mathrm{mg}$. Para la monensina solo fue posible detectar el compuesto hasta las 4 horas en una vaca tratada con $300 \mathrm{mg}$. Para esta experiencia debe tenerse presente que el líquido ruminal representa una matriz heterogénea, lo que dificulta conocer con certeza cuánto tiempo pueden permanecer estos compuestos en el rumen, por los problemas generados por interferentes consumidos al momento del análisis.

Para el estudio sobre las muestras de leche, en el caso de la lasalocida, ninguna de las 162 muestras de leche de los animales tratados como en las 36 muestras de los animales controles fue posible detectar la lasalocida (LD: $30 \mathrm{ng} / \mathrm{mL}$ ) utilizando el método analítico por HPLC.

De igual manera, para la monensina, en ninguna de las 189 muestras de leche de los animales tratados como en las 21 muestras de los animales controles fue posible detectar monensina sódica (LD: $5 \mathrm{ng} / \mathrm{mL}$ ) utilizando el método analítico por HPLC.

Cuadro 1. Condiciones de análisis cromatográfico de los ionóforos lasalocida y monensina.

Chromatographic conditions for analysis of lasalocid and monensin.

\begin{tabular}{|c|c|c|}
\hline Condición compuesto & Lasalocida & Monensina \\
\hline Longitud de onda & $315 \mathrm{~nm}$ & $520 \mathrm{~nm}$ \\
\hline Fase móvil & Metanol:Agua (90:10) & Metanol:Acetonitrilo:Agua (64:30:6) \\
\hline Temperatura de horno & $25^{\circ} \mathrm{C}$ & $40^{\circ} \mathrm{C}$ \\
\hline Flujo bomba A & $1,2 \mathrm{~mL} / \mathrm{min}$ & $0,8 \mathrm{~mL} / \mathrm{min}$ \\
\hline Flujo bomba B & No aplica & $0,8 \mathrm{~mL} / \mathrm{min}$ \\
\hline Inyección & $100 \mu \mathrm{L}$ & $100 \mu \mathrm{L}$ \\
\hline Agente derivatizante & No aplica & Metanol: $\mathrm{H}_{2} \mathrm{SO}_{4}$ : Vainillina $(950: 20: 30) 98^{\circ} \mathrm{C}$ \\
\hline
\end{tabular}


Cuadro 2. Muestras de leche negativas y positivas a la detección mediante técnica HPLC de los ionóforos lasalocida (LD: 0,03 $\mu \mathrm{g} / \mathrm{mL}$ ) y monensina (LD: $0,005 \mu \mathrm{g} / \mathrm{mL}$ ) obtenidas de vacas suplementadas con estos productos.

Positive and negative milk samples to detection through HPLC technique of the ionophores lasalocid (DL: $0,03 \mu \mathrm{g} / \mathrm{mL}$ ) and monensin (DL: $0,005 \mu \mathrm{g} / \mathrm{mL}$ ) from cows supplemented with these products.

\begin{tabular}{lcclccc}
\hline & \multicolumn{2}{c}{ Lasalocida } & & \multicolumn{2}{c}{ Monensina } \\
\cline { 2 - 3 } \cline { 5 - 6 } & Tratados & Controles & & Tratados & Controles \\
\hline Negativos & 162 & 36 & & 189 & 21 \\
Positivos & ND & ND & & ND & ND \\
\hline
\end{tabular}

ND: No detectado.

El resumen de los resultados obtenidos para los ionóforos del estudio se presenta en el cuadro 2.

Para explicar los resultados en leche debe tenerse presente que los ionóforos lasalocida y monensina son poliéteres altamente lipofílicos que se acumulan en membranas celulares y catalizan un rápido movimiento de iones (Pressman 1976). Ellos actúan como antiportadores al ligar protones o iones metálicos (ej. sodio, potasio, calcio, magnesio) y moverlos por las membranas celulares. Debido a que la membrana celular es de naturaleza hidrofóbica, solo los ionóforos no cargados, es decir, aquellos que se encuentren protonados o coordinando un ión metálico, pueden moverse libremente por ella. Los ionóforos monensina y lasalocida a menudo son más efectivos cuando el $\mathrm{pH}$ es bajo, debido a que su grupo carboxilo se encuentra protonado con mayor probabilidad (Chow y Russell 1990) y podrá alterar la gradiente iónica. Si el pH es más alto que el pKa de la molécula, el ionóforo se encontrará ionizado, disminuyendo así su capacidad para alterar gradientes iónicas.

Las propiedades básicas de interés de los ionóforos son valores de $\mathrm{pKa}$, solubilidad en agua, coeficiente de partición octanol/agua (Kow) y su probable formación de complejos con metales o con componentes orgánicos. Estos compuestos son conocidos por formar complejos con metales alcalinos cuando el $\mathrm{pH}>\mathrm{pKa}$ (Hansen y col 2009). Estos compuestos pueden formar complejos catiónicos cuando se encuentran deprotonados, obteniéndose de este modo complejos neutros (Pressmann 1976, Elliott y col 1998). Los valores de pKa para estos ionóforos son de 2,76 y 4,75 para lasalocida y monensina, respectivamente, estimados en medio acuoso (Cox y Van Truong 1984).

$\mathrm{El} \mathrm{pH}$ de la leche bovina se caracteriza por ser ligeramente ácido, con valores que van de 6,6 hasta 6,8 (Wilson y col 1980, Park y col 2007). Particularmente, para las vacas utilizadas en este estudio, la cantidad de proteínas se encontró dentro del rango de 2,83 a 3,86\% (promedio $3,38 \%$ ), el porcentaje de grasa varió dentro del rango de 2,88 a $5,0 \%$ (promedio $3,97 \%$ ) y la producción de leche se encontró entre 13,3 y 34,7 litros/vaca/día (promedio 22,9 litros/vaca/día) para el periodo del muestreo ${ }^{1}$.

El paso de fármacos desde el plasma hacia la leche ha sido estudiado extensamente en cabras y vacas (Rasmussen 1961, Miller y col 1967). Las razones de concentración de fármaco no unido a proteínas entre leche y plasma se encuentran estrechamente relacionadas con valores calculados sobre la base de la teoría de partición por $\mathrm{pH}$. Son independientes de la concentración y son consistentes con la transferencia de fármacos desde el plasma a la leche por difusión pasiva de la fracción de fármaco no ionizada y no unida a proteínas (Rasmussen 1961). Debido al pH de la leche bovina y el de la sangre $(7,4)$, el cociente leche/ plasma será mayor para los fármacos básicos, similar para los neutros y menor para los ácidos.

Otro factor relacionado con lo antes presentado y que permite estimar si un fármaco pasará a la leche es el grado de ionización en plasma. Debe tenerse presente que el grado de no ionización para un fármaco se relaciona directamente con la cantidad de fármaco disponible para atravesar membranas biológicas, lo que ha sido ampliamente estudiado en caprinos y bovinos (Davson y Danielli 1943, Rasmussen 1973).

Al evaluar el grado de ionización de estas moléculas en plasma mediante aproximaciones de la ecuación de Henderson Haselbalch se obtienen valores de no ionizado/ ionizado de $2,2908 \times 10^{-5}$ para lasalocida y $2,2387 \times 10^{-3}$ para monensina, lo que significa que estos compuestos se encontrarán ionizados en plasma en 99,99\% y 99,97\%, respectivamente.

Se concluye que los ionóforos lasalocida y monesina administrados en vacas lecheras en la dosis recomendada por el fabricante no son posibles de detectar en muestras de leche cruda con un límite de detección de $30 \mathrm{ng} / \mathrm{mL}$ o ppb y $5 \mathrm{ng} / \mathrm{mL}$ o ppb, respectivamente.

La técnica utilizada para la monensina cumple con la normativa en cuanto a la presencia de residuos de Canadá (MRL 10 ppb), Japón (MRL10 ppb) y USA (no reportados), pero no lo hace respecto a la de la EMA (MRL 2 ppb).

\section{REFERENCIAS}

Bergen WG, DB Bates. 1984. Ionophores: their effect on production efficiency and mode of action. J Anim Sci 58, 1465-1483.

Chow JM, JB Russell. 1990. Effect of ionophores and $\mathrm{pH}$ on growth of Streptococcus bovis in batch and continuous culture. Appl Environ Microbiol 56, 1588-1593.

Cox B, N van Truong. 1984. Stability constants of complexes of monensin and lasalocid with alkali-metal and alkaline-earth-metal ions in protic and polar aprotic solvents. J Chem Soc, Faraday trans 1. 80, 3275-3284.

Davson H, JF Danielli. 1943. The permeability of natural membranes. Cambridge University Press, London,UK.

Duffield TF, RN Bagg. 2000. Use of ionophores in lactating dairy cattle: A review. Can Vet J 41, 388-394.

1 Información del Control Lechero de Cooprinsem, Chile. 
Elliot CT, DG Kennedy, WJ McCaughey. 1998. Rapid screening for monensin residues in poultry plasma by a dry reagent dissociation enhanced lanthanide fluoroimmunoassay. Analyst 123, 2493-2496.

Espíndola MS. 2010. Alternativas que permiten modificar la fermentación ruminal en vacas lecheras. En: Contreras P, Noro M (eds). Rumen: morfofisiología, trastornos y modulación de la actividad fermentativa. $3^{\mathrm{a}}$ ed. Imprenta América, Valdivia, Chile, Pp 119-126.

FDA, Food and Drug Administration. 2001. Guidance for Industry: Bioanalytical Method Validation. FDA, USA, Pp 1-22.

Gandra JR, FP Rennó, JE Freitas Júnior, M Maturana Filho, RV Barletta. 2012. Nutrients balances and milk fatty acids profile of mild lactation dairy cows supplemented with monensin. Rev Bras Saúde Prod Anim, Salvador 13, 1180-1196.

Gustafson RH. 1991. Use of antibiotics in livestock and human health concerns. J Dairy Sci 74, 1428-1432.

Hansen M, E Björklund, KA Krogh, B Halling-Sorensen. 2009. Analytical strategies for assessing ionophores in the environment. Trends Analyt Chem 28, 521-33.

Jerez A, R Chihuailaf, M Gai, M Noro, F Wittwer. 2013. Development and validation of a HPLC method to determine lasalocid in raw milk samples from dairy cows. Revista Científica, FCV-LUZ 23, 537-542.

McGuffey RK, LF Richardson, JID Wilkinson. 2001. Ionophores for dairy cattle: Current status and future outlook. J Dairy Sci 84 (E-Suppl.), E194-E203.

Miller GE, NC Banerjee, CM Stowe. 1967. Diffusion of certain weak organic acids and bases across the bovine mammary gland membrane after systemic administration. J Pharmacol Exp Ther 157, 245-253.

Mohamadi MC, ADF Shahraki, A Pirestani, GR Ghalamkari, AR Ghasrodashti. 2009. Effects of monensin on some of the metabolic hormones and ketone bodies in transition dairy cows. Analele IBNA 25, 91-97.

Park YW, M Juárez, M Ramos, GFW Haenlein. 2007. Physico-chemical characteristics of goat and sheep milk. Small Ruminant Res 68, 88-113.

Pinos Rodríguez JM, SS González Muñoz. 2000. Efectos biológicos y productivos de los ionóforos en rumiantes. Interciencia 25, 379-385.

Pressman BC. 1968. Ionophorous antibiotics as models for biological transport. Fed Proc 27, 1283-1288.

Pressman BC. 1976. Biological applications of ionophores. Annu Rev Biochem 45, 501-530.

Rasmussen F. 1961. Mammary excretion of antipyrine, ethanol, and urea. Acta Vet Scand 2, 151-156.

Rasmussen F. 1973. The mechanism of drug secretion into milk. In: Galli C (ed). Dietary lipids and postnatal development. Raven Press, New York, USA, Pp 231-245.

Russell JB. 1987. A proposed mechanism of monensin action in inhibiting ruminal bacterial growth: effects on ion flux and protonmotive force. J Anim Sci 64, 1519-1525.

Russell JB, HJ Strobel, G Chen. 1988. Enrichment and isolation of a ruminal bacterium with a very high specific activity of ammonia production. Appl Environ Microbiol 54, 872-877.

Russell JB, HJ Strobel. 1989. Effect of ionophores on ruminal fermentation. Appl Environ Microbiol 55, 1-6.

Russell JB, H Mantobani. 2002. The bacteriocins of ruminal bacteria and their potential as an alternative to antibiotics. J Mol Microbiol Biotechnol 4, 347-355.

Wilson JT, RD Brown, DR Cherek, JW Dailey, B Hilman, PC Jobe, BR Manno, JE Manno, HM Redetzki, JJ Stewart. 1980. Drug excretion in human breast milk: Principles, pharmacokinetics and projected consequences. Clin Pharmacokinet 5, 1-66. 